\title{
DISTRIBUIÇÃO DO PARTO VAGINAL E DA CESARIANA NO MUNICÍPIO DO RIO DE J ANEIRO NO PERÍODO DE 2001 A 2004
}

\author{
Distribution of the Vaginal Birth and of the Cesarean \\ in the City of Rio de J aneiro from 2001 to 2004 \\ Distribución del Parto Vaginal y de la Cesarea en el Municipio \\ del Rio de J aneiro en el Periodo de 2001 hasta 2004
}

Virginia Maria de Azevedo Oliveira Knupp ${ }^{1} \quad$ Enirtes Caetano Prates Melo²

Rejane Burlandi de Oliveira ${ }^{3}$

\begin{abstract}
Resumo
0 parto vaginal é um processo natural que acontece por si mesmo, não necessitando da intervenção de terceiro. A cesariana é uma intervenção médica sobre um processo que a natureza resolve e regula sem artifícios. 0 objetivo desse estudo é analisar a distribuição do par to vaginal e da cesariana no Município do Rio de Janeiro no período de 2001 a 2004 e estudálos segundo as variáveis: raça da parturiente, área de planejamento de residência, local de ocorrência, natureza jurídica do hospital, faixa etária, estado civil e escolaridade da mãe, consulta pré-natal e Apgar no $1^{\circ}$ minuto. Estudo descritivo de base populacional, que analisou as Declarações de Nascidos Vivos do Município do Rio de Janeiro em 2001 a 2004 através do Sistema de Informações sobre Nascidos Vivos. As altas taxas de cesariana foram observadas nas regiões com maior poder econômico, instituições privadas, em mulheres acima de 35 anos, em casadas e com maior escolaridade.
\end{abstract}

Palavras-chave: Enfermagem Obstétrica. Saúde da Mulher. Sistemas de Informação. Saúde Coletiva.

\begin{abstract}
The vaginal birth is a natural process that happens for it self, no needing other's intervention. The cesarean is a medical intervention above a process that the nature resolves itself without artifices. The objective of this study is to analyze the distribution of the vaginal birth and of the cesarean in the City of Rio de Janeiro from 2001 to 2004 and to study it according the variables: race of the parturiente, area of the planning of the residence, place of occurrence, juridical nature of the hospital, age group, marital status and mother education, prenatal consultation and Apgar in the first minute. Descriptive study, of populational basis, which analyzed the Declarations of the Living Born in the City of Rio de Janeiro in 2001 to 2004 trough the Information System about Living Born. The high rate of cesareans were observed in the regions with higher economic status, private institutions, in women above 35 years old, married and with high education.
\end{abstract}

Keywords: Obstetrical Nursing. Women's health. Information Systems. Public Health.

\section{Resumen}

El parto vaginal es un proceso que acontece por si mismo, no habiendo necesidad de intervención de terceros. La cesarea es una intervención médica sobre un proceso que la naturaleza resolve sin artificios. El objetivo de este estudio es analizar la distribución del parto vaginal y de la cesarea en el Municipio del Rio de Janeiro en el periodo de 2001 hasta 2004 y estudiar según las variables: raza de la parturiente, área de planificación de residencia, ubicación de ocurrencia, naturaleza jurídica del hospital,edad, estado civil y escolaridad de la madre, consulta prenatal y Apgar en el primer minuto. Estudio descriptivo de base poblacional, que analisó las Declaraciones de Nacidos Vivos del Municipio del Rio de Janeiro en 2001 hasta 2004 a través del Sistema de Información sobre Nacidos Vivos. Las altas tazas de cesarea fueron observadas en las regiones con mayor poder económico, instituciones privadas, en mujeres con más de 35 años, en casadas y con mayor escolaridad.

Palabras clave: Enfermería Obstétrica. Salud de la Mujer. Sistemas de Información. Salud Pública. 


\section{INTRODUÇÃO}

Estudos sobre o parto vaginal e a cesariana têm sido aplicados aos diversos problemas associados à organização da assistência prestada a mulher durante a gravidez, o parto e 0 puerpério ${ }^{1 ; 2}$. Tais problemas emergiram com 0 desenvolvimento de um modelo de assistência obstétrica voltado para as necessidades sociais, com a manutenção do crescimento populacional saudável e a redução das altas taxas de mortalidade infantil, mas sem atender as necessidades de saúde específicas da população feminina ${ }^{3}$.

Esse modelo foi caracterizado pela alteração da posição adotada pela gestante durante 0 trabalho de parto, que passou de vertical para horizontal, fazendo com que a mulher assumisse uma postura submissa e dependente em relação aos profissionais de saúde, que passaram a ter pleno domínio das questões ligadas ao nascimento. Além disso, o parto deixou de ser doméstico (privado) e fisiológico, passando a ser institucionalizado (público) e patológico (medicalização do parto), sendo um fator determinante para afastar a família do processo do nascimento, visto que a estrutura física e as rotinas hospitalares foram desenvolvidas para atender as necessidades dos profissionais de saúde, e não das gestantes ${ }^{4 ; 5}$.

Diante dessa realidade, o par to passou a ser visto como um "fenômeno médico" pautado nas intervenções cirúrgicas, como a cesariana, e no modelo curativo. A década de 1970 foi marcada pelo aumento indiscriminado do número de cesarianas em função de vários fatores, como a organização da assistência obstétrica, a maior remuneração da cesárea, a associação da laqueadura tubária e a participação da mulher na escolha do tipo de parto ${ }^{6}$.

Em 1985 a Organização Mundial de Saúde (OMS) estabeleceu que a taxa máxima de cesariana seria de $15 \%$. No entanto, apesar da existência deste limite, vários estudos mostram que a taxa de cesárea permanece elevada estando muito acima deste dos padrões recomendados ${ }^{1 ; 2}$. Essa situação é em parte decorrente da banalização da cesárea associada ao caráter comercial que assumiu nos últimos anos, além dos problemas encontrados na formação do profissional de saúde e questões ligadas à gestante, como o medo da dor do parto normal, da deformação do corpo e influência na vida sexual, como a perda do prazer em função das alterações ocorridas durante o parto ${ }^{7}$. Há que se reconhecer ainda que 0 aprimoramento da técnica cirúrgica e anestésica favorece o parto programado.

0 Ministério da Saúde 1 , para tentar reduzir as altas taxas de cesariana, estabeleceu um limite de $40 \%$ nas cesáreas realizadas através do Sistema Único de Saúde (SUS) em cada região, deixando de pagar a conta hospitalar do excedente. Foram tomadas ainda algumas medidas adicionais, dentre elas a realização de cursos e seminários para a qualificação profissional, com o objetivo de rever as práticas adotadas e incentivar o parto normal e a humanização do parto; também tem sido estimulado o curso para enfermeiras obstetras ( 1,5 mil profissionais já foram qualificados), e 350 doulas (mulheres que dão suporte físico e emocional a outras mulheres antes, durante e após o parto) foram treinadas ${ }^{8}$.
A cesariana apresenta riscos inerentes ao ato cirúrgico, além de aumentar o tempo de recuperação da puérpera e influenciar no aumento da taxa de mortalidade materna e neonatal, uma vez que a chance da mulher morrer na cesariana é seis vezes maior que no parto normal, principalmente considerando que a possibilidade de contrair uma infecção ou ter uma hemorragia é muito maior. Em relação ao recém-nascido, o risco de ir para Unidade de Terapia Intensiva quadruplica entre os nascidos por cesariana, que ao ser marcada não leva em consideração a maturidade do feto, principalmente do pulmão, sendo realizada independentemente do início do trabalho de parto8.

Apesar destes aspectos negativos, a cesariana é indicada com a intenção de salvar a vida da mãe e do filho em situação de alto risco, tais como: sofrimento fetal, apresentação pélvica, hemorragia antes do parto, doença hipertensiva específica da gravidez (DHEG), gemelaridade, diabetes e cesárea de repetição $0^{9}$. A indicação da cesariana em gestantes portadoras do vírus HIV tornou-se uma forma segura de evitar a transmissão do recém-nascido em até $90 \%{ }^{10}$.

Uma questão fundamental para o planejamento do setor saúde é a distribuição espacial dos serviços e de sua clientela. Os serviços de saúde estão inevitavelmente concentrados em determinados lugares e, conseqüentemente, são mais acessíveis às pessoas que deles estão mais próximas do que àquelas que estão mais distantes deles. Desigualdades no acesso à assistência de qualidade têm papel fundamental na determinação da mortalidade materna e infantil, e a possibilidade de intervenção no perfil da mortalidade nesse grupo específico desloca-se, cada vez mais, para a esfera dos serviços de saúde. Esse estudo tem como objetivo analisar a distribuição do parto vaginal e da cesariana no Município do Rio de Janeiro no período de 2001 a 2004.

\section{METODOLOGIA}

Trata-se de um estudo descritivo de base populacional que analisou as declarações de nascidos vivos do Município do Rio de Janeiro, no período de 2001 a 2004. Para a realização do estudo foram utilizados os dados do Sistema de Informações sobre Nascidos Vivos (SINASC), disponibilizados pela Secretaria Municipal de Saúde do Rio de Janeiro após aprovação do estudo pelo Comitê de Ética em Pesquisa do Hospital Universitário Gaffrée e Guinle.

O SINASC tem como objetivo propiciar a obtenção regular de dados sobre os nascidos vivos, de forma abrangente e confiável, que possa embasar os diversos níveis de gerenciamento em suas ações de saúde. 0 instrumento de coleta é a Declaração de Nascidos Vivos (DNV), instrumento padronizado e impresso pelo Ministério da Saúde ${ }^{11}$.

Incluiu-se no estudo a análise do parto vaginal e da cesariana segundo as variáveis: raça da parturiente (branca, negra, amarela, parda e indígena), área de planejamento de residência, local de ocorrência do nascimento (hospital, outro estabelecimento de saúde e domicílio), natureza jurídica do hospital (SUS e não-SUS - privado), faixa etária da mãe ( $<14$ anos, 15 a 19 anos, 20 a 34 anos, 35 anos ou mais), estado civil (solteira, casada/união consensual, viúva e separada 
judicialmente), escolaridade da mãe em anos (nenhuma, 01 a 03, 04 a 07, 08 a 11, 12 anos ou mais), número de consultas durante o pré-natal (nenhuma, 1 a 3 vezes, 4 a 6 vezes, 7 consultas e mais) e índice de Apgar no $1^{\circ}$. minuto ( 0 a 3, 4 a 7 e 8 a 10). Os estratos de cada variável foram utilizados nos cálculos e nas representações gráficas.

A área de estudo é o Município do Rio de Janeiro, administrativamente dividido em 153 bairros, que se encontram agregados em 30 Regiões Administrativas (RA). As RAs compõem cinco Áreas de Planejamento (AP); as APs 2, 3 e 5 foram posteriormente subdivididas, totalizando dez áreas. Estas áreas mantêm em comum, além da proximidade geográfica, o perfil socioeconômico e de acesso aos serviços de saúde. A AP 1 configura a zona mais antiga e central da cidade. As APs 2.1 e 2.2 correspondem respectivamente à Zona Sul e Zona Norte. As APs 3.1, 3.2 e 3.3 correspondem ao subúrbio da Central e Leopoldina, além da llha do Governador. As APs 4, 5.1, 5.2 e 5.3 correspondem à Zona Oeste.

0 georreferenciamento foi efetuado com base na variável bairro de residência, que consta na DNV. 0 processamento dos dados e o mapeamento foram feitos através do TAB para Windows - TabWin (Datasus). 0 TAB para Windows, programa de código aberto desenvolvido pelo Datasus (www.datasus.gov.br), está disponível gratuitamente a todo e qualquer interessado. Trata-se de um aplicativo que permite tabular informações de diferentes tipos (dados de internação hospitalar, de mortalidade, de população, etc.) em um mesmo ambiente. A análise dos dados foi realizada através do cálculo direto das medidas de freqüência.

\section{RESULTADOS}

No ano de 2004 os nascimentos ocorridos no Município do Rio de Janeiro estavam distribuídos em 93 hospitais, dos quais 30 vinculados ao Sistema Único de Saúde - SUS (Figura 1).

Figura 1:

Distribuição dos hospitais com nascimento em 2004, segundo RA e vinculação ao Sistema Único de Saúde, Município do Rio de Janeiro.

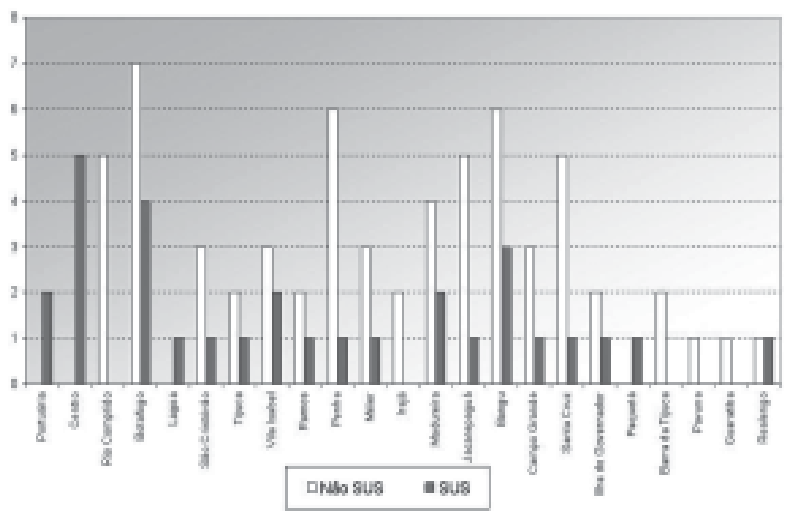

0 Sistema de Informações sobre Nascidos Vivos registrou 347.255 nascimentos no Município do Rio de Janeiro no período analisado, dos quais 165.905 correspondiam à cesariana. Observou-se uma queda do número deste procedimento em 2002 (46,5\%) e um discreto aumento em 2004 (49,4\%) (Figura 2).
Figura 2:

Distribuição proporcional dos partos vaginal e cesáreo no Município do Rio de Janeiro, 2001 a 2004.

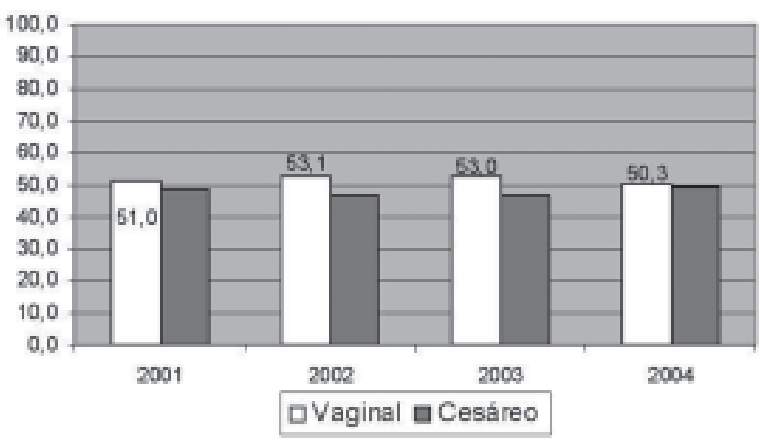

Do total de nascimentos registrados, 342.507 (98,6\%) ocorreram no hospital. Dos nascimentos ocorridos na rede do SUS, verificou-se que $67,9 \%$ correspondiam ao parto vaginal, e, nos da rede privada, 83,2\% correspondiam à cesariana.

\section{Figura 3:}

Distribuição espacial do tipo de parto (vaginal) segundo AP de residência no Município do Rio de Janeiro no período de 2001 a 2004.
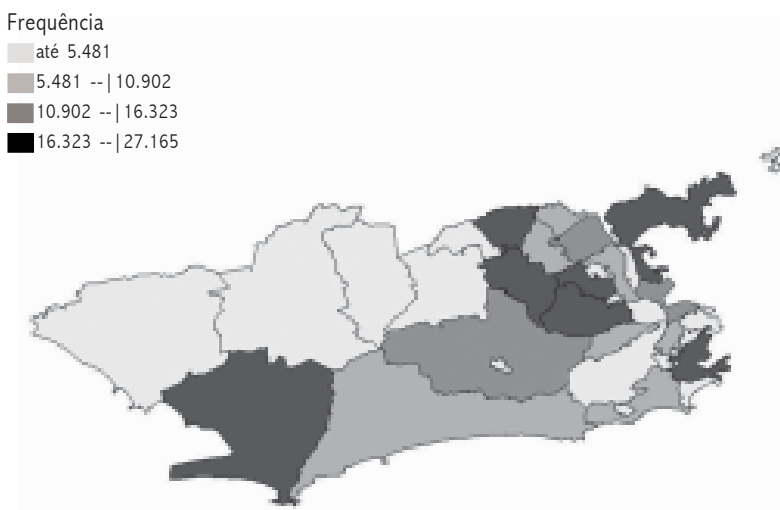

Ao analisar o parto vaginal e a cesariana segundo a AP de residência da mãe, verificou-se uma distribuição heterogênea do parto vaginal (Figura 3). Este padrão não se mantém nas mulheres submetidas à cesariana, apresentando maior concentração na Zona Sul (AP 2.1), com destaque para a RA de Botafogo (Figura 4).

\section{Figura 4:}

Distribuição espacial do tipo de parto (cesáreo) segundo AP de residência no Município do Rio de Janeiro no período de 2001 a 2004.

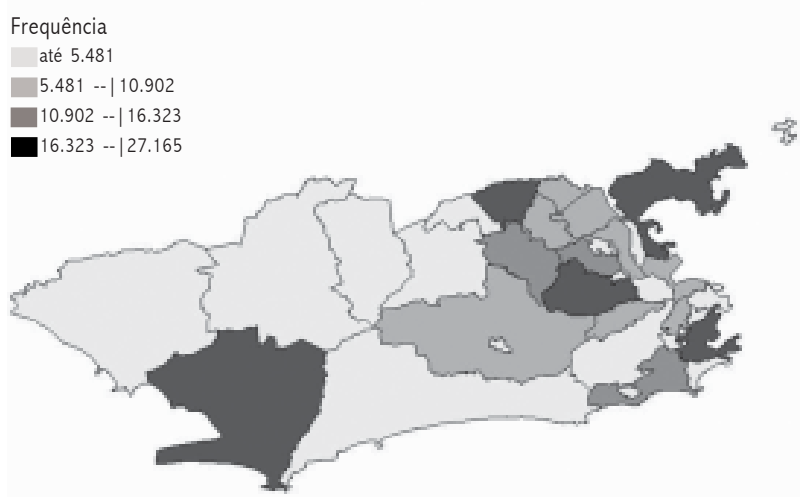


Verificou-se um predomínio da taxa de cesariana na raça/etnia branca $(55,1 \%)$. No parto vaginal, as maiores taxas foram observadas entre mulheres negras $(66,4 \%)$. Comportamento semelhante foi observado entre mulheres pardas. Já as raças amarela e indígena, com freqüências muito menos expressivas, não apresentaram um padrão que mereça destaque.

A distribuição por faixa etária revelou uma associação com a cesariana em que o aumento da idade é diretamente proporcional com as taxas de cesariana, ou seja, quanto maior a idade da mãe maior a taxa observada. Nas mulheres com 35 anos ou mais, a taxa de cesariana foi de $64,2 \%$. Em relação ao parto vaginal, as maiores taxas foram encontradas nas mulheres na faixa etária entre 15 e 19 anos (71,6\%).

Quanto ao estado civil, a maior taxa de cesariana foi observada entre mulheres casadas/em união consensual $(59,5 \%)$, enquanto a do parto vaginal foi maior entre mulheres solteiras $(61,2 \%)$.

Foi observado um gradiente entre a escolaridade da gestante e as taxas de cesariana estratificadas. Nessa distribuição, quanto maior a escolaridade maior a taxa de cesariana, com maior destaque que para aquelas com 12 anos ou mais anos de estudo, com uma taxa $68,2 \%$. Já a taxa de parto vaginal apresentou um comportamento inverso, com $74,6 \%$ de cesáreas em mulheres com nenhuma escolaridade) (Figura 5).

Figura 5:

Distribuição proporcional da escolaridade da mãe segundo os partos vaginal e cesáreo no Município do Rio de Janeiro, 2001 - 2004.

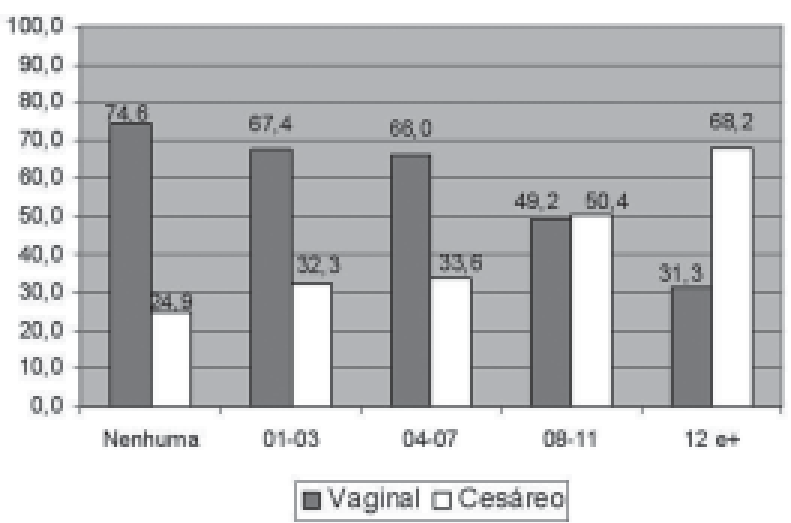

Ao analisar o número de consultas durante o prénatal, verificou-se que, do total de mulheres que não foram a nenhuma consulta, $80,2 \%$ foram submetidas ao parto vaginal. Seguindo tendência inversa, a taxa de cesariana apresentou um crescimento proporcional ao número de consultas. As maiores taxas de cesariana foram registradas entre mulheres que freqüentaram 7 ou mais consultas durante o pré-natal $(57,6 \%)$ (Figura 6).

Em relação ao Apgar no $1^{\circ}$ minuto, não se observou um comportamento definido na distribuição dos partos vaginais e das cesarianas.
Figura 6:

Proporção de consulta pré-natal segundo o tipo de parto no Município do Rio de Janeiro, 2001 - 2004.

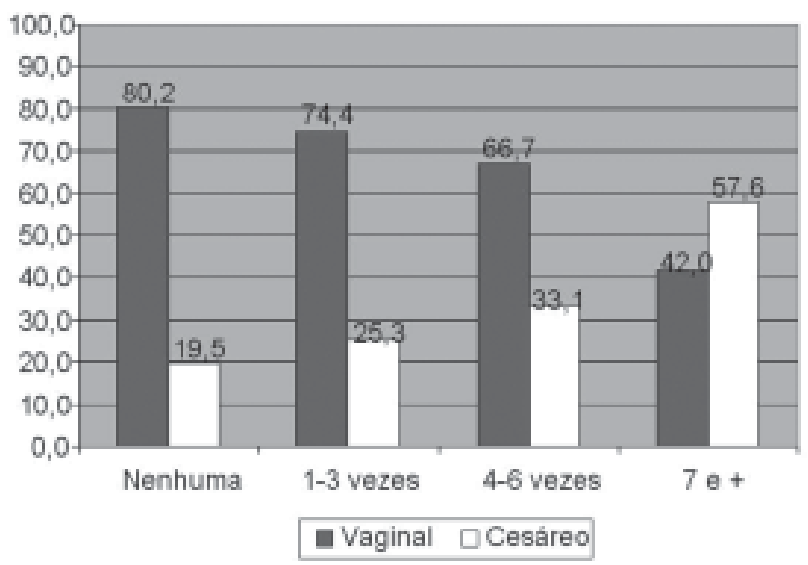

\section{DISCUSSÃO}

A alta concentração de cesariana na rede privada pode ser explicada em parte pelo caráter comercial atribuído a esta prática. Vale destacar que 0 atendimento no setor público se dá, principalmente, em maternidades vinculadas à esfera municipal, quando dificilmente se estabelece um vínculo entre a gestante e o sistema de saúde. A impossibilidade da garantia da vaga no estabelecimento de saúde onde a gestante realizou o pré-natal ocasiona o processo de peregrinação das gestantes entre as maternidades em busca de atendimento. No setor privado verifica-se uma tendência inversa, a mulher é acompanhada durante a gestação pelo médico que realizará o seu parto, tendo a oportunidade de estabelecer uma relação prévia de confiança, o que, entretanto, não garante uma condução mais adequada do parto ${ }^{7}$.

A análise da variável local de ocorrência suscita a necessidade de uma reflexão sobre as questões que envolvem 0 acesso às maternidades, visto que quase $100 \%$ dos partos no Município do Rio de Janeiro ocorreram nos hospitais. A acessibilidade deve ser garantida segundo o enfoque geográfico (localização dos serviços de saúde), funcional (oferta dos serviços de saúde), econômico e cultural (adequação dos serviços aos hábitos e costumes da população a ser atendida) ${ }^{9}$.

A distribuição espacial do parto vaginal e da cesariana não mantém relação com o local de residência da gestante, mas com a localização dos estabelecimentos de saúde que prestam assistência obstétrica e com a natureza jurídica dos hospitais (SUS e não-SUS - privado). Os hospitais privados concentramse principalmente na Zona Sul (AP 2.1), principalmente em Laranjeiras e Humaitá. Os hospitais particulares conveniados ao SUS localizam-se predominantemente no centro da cidade, Jacarepaguá, Olaria, Penha Circular e na Zona Oeste.

0 padrão mais heterogêneo observado na análise espacial do parto vaginal pode ser explicado pela distribuição dos estabelecimentos públicos de saúde no Município do Rio de Janeiro. Os hospitais estaduais estão localizados na Zona Oeste e na AP 3.1 (Penha Circular); e os hospitais universitários, na Cidade Universitária, Tijuca e Vila Isabel. Esses hospitais gerais apresentam um número reduzido de leitos obstétricos. Os 
hospitais federais concentram-se na região central da cidade e em Bonsucesso. Já os hospitais municipais que prestam assistência obstétrica e dispõem do maior número de leitos se concentram na área central da cidade (Centro, São Cristóvão) e na Zona Norte (Lins, Madureira, Marechal Hermes).

A variável raça/etnia merece uma análise mais cuidadosa em função da limitação relativa à qualidade dos dados. A formulação atual da raça/etnia, no que se refere à saúde, utiliza como indicador mais freqüente a cor da pele. No entanto, trata-se de uma definição muito abrangente do que simplesmente a "cor da pele". Formas de identificação racial, étnica ou por ancestralidade não são suscetíveis aos critériospadrão de cientificidade, dada a possibilidade de os indivíduos mudarem sua identidade étnico-racial ao longo do tempo e em decorrência de circunstâncias políticas e sociais ${ }^{12}$.

A alta concentração de cesarianas entre mulheres com mais de 35 anos pode estar relacionada ao fato de estas gestantes possuírem maior risco para algumas patologias graves relacionadas à gravidez, tais como diabetes mellitus e doença hipertensiva específica da gestação (DHEG), que podem exigir a interrupção da gestação antes da maturação fetal ${ }^{8}$.

A taxa de cesariana em mulheres casadas deve ser analisada em conjunto com outras variáveis econômicas e sociais que, agregadas a essa informação, poderiam definir um dado padrão de risco para o desfecho estudado (o parto). Um estudo sobre a importância do acompanhante identificou uma queda na taxa de cesariana, seguida pela redução do uso de ocitocina, duração do trabalho de parto e efeitos em médio prazo, como a redução dos casos de depressão pós-parto ${ }^{8}$. Mas a presença de um acompanhante, mesmo que escolhido pela mulher, por si só não pode ser considerado como sinônimo de suporte ${ }^{4}$.

0 aumento da taxa de cesariana verificado entre as mulheres com maior escolaridade pode estar associado a questões de caráter econômico e ligadas ao acesso aos serviços de saúde. Primeiro pela crescente presença da mulher no mercado de trabalho, que opta pela cesariana em função de uma possível facilidade em determinar a data e a hora para sua realização, não comprometendo suas atividades profissionais $^{12}$. Seguido pelo aumento do poder aquisitivo dessas mulheres, que favorece o acesso e possibilita a compra de bens materiais de toda ordem.

Considerando que quase $90 \%$ das mulheres fazem prénatal com profissionais de saúde formalmente qualificados, enfermeiros ou médicos, e que a média de consultas no SUS está acima de quatro por gestante, o Brasil já deveria apresentar melhores indicadores de saúde materna e perinatal.

\section{Referências}

1. Hotimsky SN, Rattner D, Venancio SI, Bógus CM, Miranda MM. 0 parto como eu vejo...ou como eu desejo? Expectativas de gestantes, usuárias do SUS, acerca do parto e da assistência obstétrica. Cad Saude Publica 2002 out; 18 (5): 1303-311.

2. Dias MAB, Deslandes SF. Cesarianas: percepção do risco e sua indicação pelo obstetra em uma maternidade pública no Município do Rio de Janeiro. Cad Saude Publica 2004 fev; 20 (1): 109-16.
Os resultados encontrados apontam para a fragilidade da assistência prestada à gestante durante o pré-natal, visto que, de um modo geral, parece que há uma maior preocupação em atingir um dado quantitativo de consultas preconizado pela OMS. Por outro lado, a qualidade dos serviços prestados ainda é um desafio a ser enfrentado. Este padrão determina uma assistência materna com sinais claros de descaso, expressos pela qualidade do registro nos prontuários, pelos cartões de acompanhamento não preenchidos, entre outros ${ }^{13}$.

0 Apgar no $1^{\circ}$ minuto não deve ser analisado apenas considerando a via de parto, já que há outros fatores que influenciam o resultado, dentre eles as co-morbidades relacionadas às gestantes e aos recém-nascidos, o tempo que o feto permanece no canal do parto, a assistência prestada ao recém-nascido, as condições da sala de parto, faixa etária da mãe, dente outros.

\section{CONCLUSÕES}

Com base neste estudo, pode-se afirmar que no decorrer dos anos analisados há uma manutenção das altas taxas de cesariana que parece manter uma associação direta com as variáveis socioeconômicas, das quais a escolaridade, a raça/etnia e o estado civil da mãe merecem destaque por atuarem como preditores de risco nas questões relacionadas ao parto e na definição de grupos prioritários para o acesso aos serviços de saúde.

A incorporação do elemento geográfico, através da sua importante contribuição na identificação de áreas e situações de risco, abre a possibilidade do redirecionamento de ações de saúde, principalmente em áreas onde se verifica maior exclusão social. Em que pese o papel do conjunto de práticas propostas para o rastreamento de condições/situações de risco durante 0 pré-natal, o parto e com o recém-nascido no nível individual, estratégias voltadas para o contexto onde o binômio mãe-bebê vive podem ser extremamente úteis.

Os resultados obtidos neste estudo constituem subsídios para uma reflexão acerca da assistência obstétrica prestada durante o pré-natal e o parto. A localização geográfica dos estabelecimentos de saúde que atendem ao parto define uma distribuição espacial dos nascimentos extremamente desigual, alternando padrões de escassez em algumas áreas e excesso em outras. A análise do perfil das mulheres submetidas à cesariana mostrou que o problema não está centrado exclusivamente na saúde, para tanto devemos considerar os aspectos sociais, culturais e econômicos que envolvem a mulher que está inserida num contexto de desigualdades.

3. Ministério da Saúde (BR). Parto, aborto e puerpério: assistência humanizada à mulher. Brasília (DF); 2001.

4. Brüggemann OM, Parpinelli MA, Osis MJD. Evidências sobre o suporte durante o trabalho de parto/parto: uma revisão de literatura. Cad Saude Publica 2005 out; 21 (5): 1316-327.

5. Silva LR, Bicchieri T. A percepção dos acadêmicos de enfermagem sobre o cuidado à mulher no trabalho de parto $\mathrm{e}$ nascimento: uma abordagem qualitativa. Esc Anna Nery R Enferm 2006 ago; 10 (2): 258-65. 
6. Mamede FV, Mamede MV, Dotto LMG. Reflexões sobra deambulação e posição materna no trabalho de parto e parto. Esc Anna Nery Rev Enferm 2007 jun; 11(2): 331-36.

7. Campos TP, Carvalho MS. Assistência ao parto no Município do Rio de Janeiro: perfil das maternidades e o acesso da clientela. Cad Saude Publica 2000 jun; 16 (2): 411-20.

8. Ministério da Saúde (BR). Saúde Brasil. 117ª ed. Brasília (DF); 2006.

9. Campos TP. Perfil de nascimentos e óbitos infantis: a busca da assistência no Município do Rio de Janeiro [dissertação de mestrado]. Rio de Janeiro (RJ): Escola Nacional de Saúde Pública/FIOCRUZ; 1997.
10. Rocco R, Leite HV, Vasconcellos M, Cabral ACV. Morbidade associada a cesariana eletiva em portadores de HIV. Rev Bras Ginecol Obstet 2003 jun; 25 (5): 323-28.

11. Secretaria de Saúde do Estado da Bahia. Diretoria de Informação e Comunicação em Saúde. SINASC/SIM. Algumas orientações. Salvador(BA);2003.

12. Laguardia J. 0 uso da variável "raça" na pesquisa em saúde. Physis: rev saude colet 2004 jul; 14 (2): 197-234.

13. Serruya SJ, Cecatti JG, Lago TG. 0 programa de humanização no pré-natal e nascimento do Ministério da Saúde no Brasil: resultados iniciais. Cad Saude Publica 2004 out; 20 (5): 1281-289. 